\title{
Publisher's Note: Inverse Ising problem in continuous time: A latent variable approach
} [Phys. Rev. E 96, 062104 (2017)]

Christian Donner and Manfred Opper

Q (Received 27 December 2017; published 3 January 2018)

DOI: 10.1103/PhysRevE.97.019901

This paper was published online on 4 December 2017 with a typographical error in the equation following Eq. (15). On page 3, the equation in the right-hand column following Eq. (15) should read as

$$
p_{\mathrm{PG}}\left(\omega_{i}^{n} \mid b, c\right)=\frac{\exp \left(-\frac{c^{2}}{2} \omega_{i}^{n}\right) p_{\mathrm{PG}}\left(\omega_{i}^{n} \mid b, 0\right)}{\cosh ^{-b}\left(\frac{c}{2}\right)},
$$

The equation has been corrected as of 27 December 2017. The equation is correct in the printed version of the journal. 